\title{
Los abejorros Bombus impatiens y Bombus ephippiatus pueden copular, pero no generan descendencia
}

Bumblebees bombus impatiens and bombus ephippiatus can copulate but do not generate offspring

Os abelhões Bombus impatiens e Bombus ephippiatus podem copular, mas não produzem descendentes

Nancy Elizabeth Ambriz Trujillo

Universidad de Guadalajara, México nancy.ambriz@cucsur.udg.mx https://orcid.org/0000-0001-5848-0746

Rosales-Rentería, R. R.

Universidad de Guadalajara, México ricardo.rosales@cucsur.udg.mx https://orcid.org/0000-0002-1645-5400

Sandoval-Legazpi, J. de J.

Universidad de Guadalajara, México slegazpi@cucsur.udg.mx https://orcid.org/0000-0003-4096-4042 


\title{
Resumen
}

El objetivo del estudio fue demostrar que bajo condiciones de laboratorio se puede producir cópula híbrida entre reina Bombus impatiens con machos Bombus ephippiatus. Un total de 25 reina Bombus impatiens fueron expuestas a 40 machos Bombus ephippiatus para posible apareamiento dentro de una caja de acrílico durante 38 días. El 8 \% (2/25) de las reinas copularon con alguno de los abejorros Bombus ephippiatus, pero ninguna de ellas generó descendencia. Una de las reinas copuladas murió a los 3 días postconcepción y la otra anidó, pero abortó en la etapa de pupa. Se concluye que en sí es posible que se presente la cópula entre reinas Bombus impatiens y machos Bombus ephippiatus, pero sin que esto genere un híbrido.

Palabras claves: cruzamiento de abejorro, etapa de pupa, nativas y desequilibrio ecológico, reinas.

\begin{abstract}
The objective of the study was to demonstrate that under laboratory conditions hybrid copula can be given between queen Bombus impatiens and males Bombus ephippiatus. A total of 25 queen Bombus impatiens were exposed to 40 Bombus ephippiatus males for possible mating within an acrylic box for 38 days. $8 \%(2 / 25)$ of the queens copulated with one of the bumblebees Bombus ephippiatus, but none of them generated offspring. One of the copulated queens died 3 days after conception and the other nested but aborted in the pupa stage. It is concluded that in it is possible that copulation occurs between queens Bombus impatiens and males Bombus ephippiatus, but without this generating a hybrid.
\end{abstract}

Keywords: bumblebee cross, pupa stage, native and ecological imbalance, queens. 


\section{Resumo}

O objetivo do estudo foi demonstrar que em condições de laboratório a cópula híbrida pode ocorrer entre a rainha Bombus impatiens com o macho Bombus ephippiatus. Um total de 25 impatiens Queen Bombus foram expostos a 40 machos de Bombus ephippiatus para possível acasalamento dentro de uma caixa de acrílico por 38 dias. 8\% (2/25) das rainhas copularam com uma das abelhas Bombus ephippiatus, mas nenhuma delas gerou descendência. Uma das rainhas copuladas morreu 3 dias após a concepção e a outra aninhada, mas abortou na fase pupal. Conclui-se que é possível que ocorra cópula entre rainhas Bombus impatiens e macho Bombus ephippiatus, mas sem que isso gere um híbrido.

Palavras-chave: zangão, estágio pupal, desequilíbrio nativo e ecológico, rainhas.

Fecha recepción: Noviembre 2019

Fecha aceptación: Julio 2020

\section{Introducción}

Las abejas Bombus son insectos sociales distribuidos en diferentes regiones de México (Dunnes, Lozier, Hines y Cameron, 2012); en total, se han diferenciado 25 especies en el país, de las cuales la más común es la Bombus ephippiatus, aunque cabe mencionar que no existen muchos estudios sobre su biología. Esta especie es una opción viable para uso comercial y para evitar la introducción de especies de abejorros no nativas que pudieran generar un desequilibrio ecológico en caso de salirse de control (Montemayor-Fuentes y Madrid-Cuevas, 2003).

Bombus ephippiatus es una especie homogénea en su estructura corporal, pero exhibe un sorprendente polimorfismo de patrón de color intraespecífico, lo que produce incertidumbre sobre sus límites genealógicos (Dunnes et al., 2012). Adicionalmente, se caracteriza por la cabeza carena en la zona temporal e hileras centrales en el pronoto que contienen entre 5 y 8 puntuaciones (Márquez, Asiain y Navarrete-Heredia, 2018). Estos abejorros están distribuidos desde el norte de México hasta el oeste de Panamá, y se encuentran por encima de los 800 m s. n. m. (Ayala y Ortega-Huerta, 2009).

Dadas estas características, tienen el potencial de emplearse como polinizadores en invernaderos, una actividad que en los últimos años ha ido en crecimiento. Así, la abundancia de este abejorro pudiera evitar la introducción de abejorros no nativos, como el Bombus 
impatiens, el cual es comercializado abundantemente por empresas transnacionales a precios bastantes elevados (Vergara y Fonseca-Buendía, 2012). Además, aun cuando se ha mencionado que estos machos de abejorro Bombus impatiens se aparean con hembras de abejorro Bombus ephippiatus, producen descendencia híbrida e infértil, y hasta el momento no se ha demostrado que la cruza pueda darse de manera inversa (Vergara y FonsecaBuendía, 2012).

La distribución de los abejorros Bombus impatiens va desde América del Norte (Canadá y Estados Unidos), desde Ontario a Maine y el sur de Florida (Yuya et al., 2009). La introducción de abejorros podría favorecer la transmisión de patógenos y la competitividad por el alimento (Morales, 2007).

Explicado lo anterior, el objetivo de este estudio es demostrar que sí hay apareamiento entre el abejorro Bombus ephippiatus y el Bombus impatiens bajo condiciones de laboratorio.

\section{Materiales y métodos}

El estudio se realizó en el laboratorio de abejorros ubicado en el Centro Universitario de la Costa Sur, Universidad de Guadalajara, con sede en la ciudad de Autlán de Navarro, Jalisco, México (Daniel Cauas, 2015). Se colocaron 25 reinas Bombus impatiens recién nacidas (Figura 1A) y 40 machos adultos Bombus ephippiatus (Figura 1B) dentro de una caja de acrílico $(32 \times 20 \times 27 \mathrm{~cm})$ durante un periodo de 38 días. Al inicio, se puso en la caja suficiente polen y jarabe para garantizar su alimentación durante el periodo de estudio. Además, estuvieron en constante monitoreo por observación directa diariamente para determinar la actividad de cortejo y monta. Se consideró cortejo cuando los machos perseguían a las reinas y lograban colocarse en la parte del dorso de ellas, mientras que se consideró que hubo cópula cuando se observó que la hembra expuso el aguijón y el macho la penetró. La variable de estudio medida fue porcentaje de hembras copulada, y se analizó dividiendo el número de hembras copuladas entre el total de hembras expuestas. 


\section{Resultados}

Se observó que solamente copularon 2 de las 25 reinas (8 \%) (figura 1C). La primera reina copuló el día 2, mientras que la otra el día 3 del periodo experimental. Ninguna de las hembras copuladas dio descendencia, ya que una de ellas murió al tercer día postconcepción sin desarrollar nido, y la otra sí desarrollo nido, pero abortó en la etapa de pupa.

Figura 1. Abejorros Bombus impatiens (imagen A), Bombus ephippiatus (imagen B) y abejorros copulando (imagen $\mathrm{C}$ )
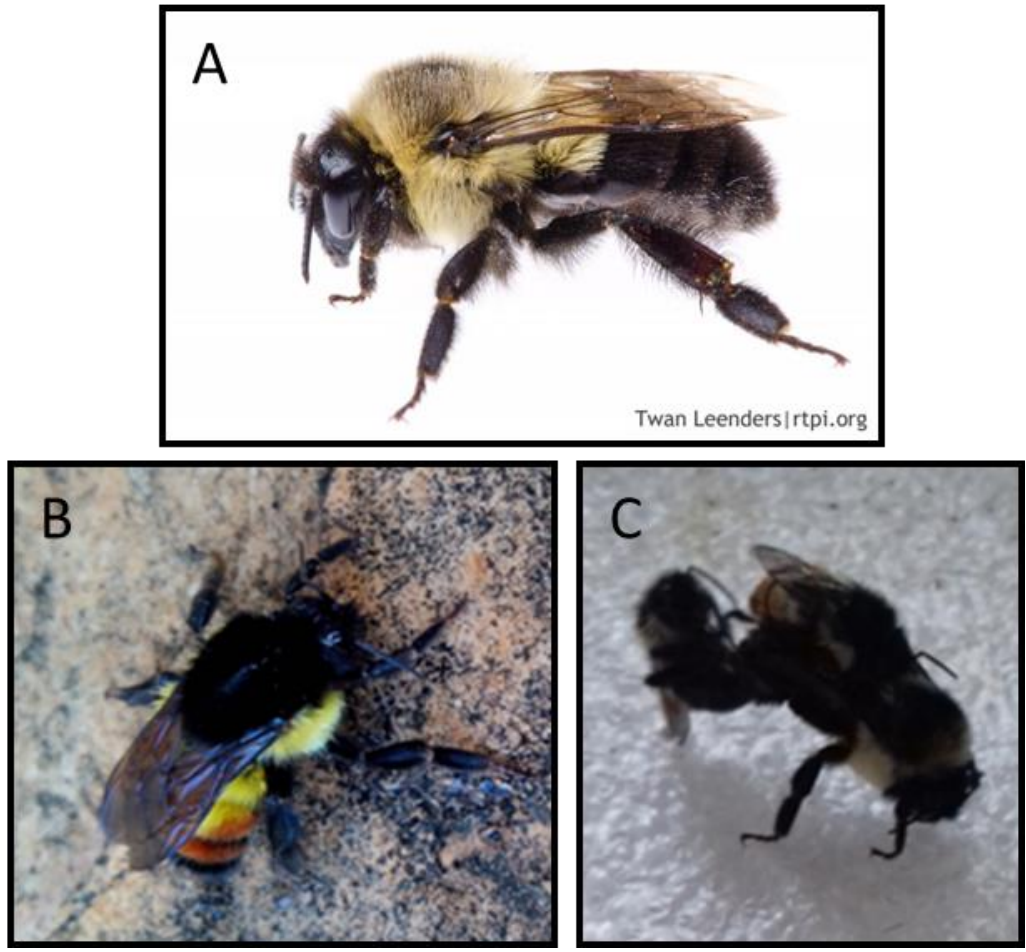

Fuente: Figura 1A Naturalista (2014); 1B Lewisboro Field Guide (2018), 1C AmbrizTrujillo (2017)

\section{Discusión}

Estos resultados coinciden con esos reportados por Yuya et al. (2009), quienes al aparear abejorros de las especies Bombus hypocrita y Bombus ignitus no obtuvieron híbridos. En otro estudio donde hicieron copulaciones interespecíficas entre Bombus terrestres y algunas especies nativas japonesas se obtuvo como resultado la producción de huevos fértiles (Yuya et al., 2009). En contraste, Hyung, et al. (2009) encontraron una descendencia de $70.3 \%$ con 
el apareamiento entre machos Bombus gynes y Bombus terrestris. En general, los resultados del presente estudio demuestran que sí existe la posibilidad de que las hembras Bombus impatiens puedan ser copuladas por machos Bombus ephippiatus.

No obstante, y aunque no se generó descendencia, se debe poner atención al respecto, ya que podría generarse un problema de reducción en la tasa reproductiva poblacional de la especie nativa (Bombus ephippiatus) por la disminución de apareamientos intraespecíficos, o incluso por una mayor mortalidad de reinas en apareamientos heteroespecíficos (Morales, 2007).

Finalmente, se destaca el hecho de la hembra copulada que abortó, lo cual podría deberse a la embriogénesis. Esto considerando que Natsuko et al. (2008) observaron embriogénesis abortada en la etapa inicial de desarrollo. Por lo tanto, tal apareamiento podría interrumpir la reproducción normal de los abejorros nativos.

\section{Conclusiones}

Los resultados encontrados en esta investigación sirven para afirmar que se culminó la cópula entre estas dos especies de abejorros Bombus impatiens y Bombus ephippiatus, y el resultado fue que de las dos reinas que copularon, una murió al tercer día y la segunda reina sí desarrollo nido, pero en la etapa de pupa abortó, por lo tanto, ninguna de ellas generó descendencia.

Esta inhibición de descendencia en estos abejorros puede tener como consecuencia futura la diminución de la especie nativa y, por ende, una falta de descendencia, lo que ocasionaría una competencia frágil por alimentación y espacio territorial entre individuos, así como la posible pérdida de la identidad de la especie por la recombinación genética entre las especies Bombus ephippiatus y Bombus impatiens. 


\section{Referencias}

Ayala, R. y Ortega-Huerta, M. (2009). El Abejorro Bombus ephippiatus Say, 1837, su Distribución Potencial y Estrategias para su manejo. Memorias del VI Congreso Mesoamericano de Abejas Nativas. Antigua, Guatemala: 165-171.

Duennes M. A., Lozier, J. D., Hines, H. M. and Cameron, S. A. (2012). Geographical patterns of genetic divergence in the widespread Mesoamerican bumble bee Bombus ephippiatus (Hymenoptera: Apidae). Molecular Phylogenetics and Evolution, 64(1), 219-231.

Cuas D. (2015). Definición de las variables, enfoque y tipo de investigación. 1-11.

Hyung, J. Y., Kim, S. Y., Lee, K. Y., Beom Lee, S., Park, I. G. and Kim1, Y I. (2009). Interspecific Hybridization of the Bumblebees Bombus ignitus and B. terrestris. Int. J. Indust. Entomol, 18(1), 41-48.

$\begin{array}{lllll}\text { Lewisboro } & \text { Field } & \text { Guide } & \text { (2018). } & \text { Retrieved }\end{array}$ https://fieldguide.lewisborolandtrust.org/bugs/eastern-bumble-bee/

Morales, C. (2007). Introducción de abejorros (Bombus) no nativos: causas, consecuencias ecológicas y perspectivas. Ecología Austral, 17, 51-65.

Montemayor-Fuentes, E. y Madrid-Cuevas, E. (2003). Biología de Bombus ephippiatus Say (Hymenoptera, Apidae) (tesis de licenciatura). Universidad de las Américas Puebla.

Natsuko, I. K., Yamanaka, D., Kanbe, Y., Kawate, Y., Yoneda, M., Tsuchida, K. y Goka, K. (2009). Reproductive disturbance of Japanese bumblebees by the introduced European bumblebee Bombus terrestris. Naturwissenschaften, 96, 467-475.

Naturalista (2014). Abejorro Mexicano (Bombus ephippiatus) observado por nationat en marzo 13. Recuperado de https://www.naturalista.mx/observations/1299336

Ambriz, T. N. E. (2017). Figura Reina Bombus impatiens copulando con macho Bombus ephippiatus.

Márquez, J., Asiain, J. y Navarrete-Heredia, J. L. (2018). Análisis taxonómico de las especies mexicanas de Belonuchus Nordmann del grupo ephippiatus (Coleoptera: Staphylinidae). Gayana, 82(1), 36-39. 
Vergara, H. y Fonseca-Buendía, P. (2012). Pollination of greenhouse tomatoes by the mexican bumblebee bombus ephippiatus (hymenoptera: apidae). Journal of Pollination Ecology, 7(4), 27-30.

Yuya, K., Ikuko, O., Masahiro, Y., Koichi, G. and Koji, T. (2009). Interspecific mating of the introduced bumblebee Bombus terrestris and the native Japanese bumblebee Bombus hypocrita sapporoensis results in inviable hybrids. Naturwissenschaften. 
Revista Iberoamericana de las Ciencias Biológicas y Agropecuarias

\begin{tabular}{|l|l|}
\hline Rol de Contribución & Autor (es) \\
\hline Conceptualización & Nancy \\
\hline Metodología & Principal Nancy y José \\
\hline Software & Ricardo \\
\hline Validación & Ricardo, igual José y Nancy principal \\
\hline Análisis Formal & NO APLICA \\
\hline Investigación & Ricardo, igual José y Nancy principal \\
\hline Recursos & Nancy \\
\hline Curación de datos & Ricardo y Nancy igual, José principal \\
\hline $\begin{array}{l}\text { Escritura - Preparación del } \\
\text { borrador original }\end{array}$ & Nancy \\
\hline $\begin{array}{l}\text { Escritura - Revisión y } \\
\text { edición }\end{array}$ & José y Nancy igual \\
\hline Visualización & José y Nancy igual \\
\hline Pupervisión & Nancy \\
\hline Adquisición de fondos & Nancy \\
\hline
\end{tabular}

